\title{
Gas sensors based on plasma-electrochemically oxidized titanium foils
}

\author{
Mhamed El Achhab and Klaus Schierbaum \\ Department of Materials Science, Institute of Experimental Condensed Matter Physics, \\ Heinrich Heine University, Düsseldorf, Germany \\ Correspondence to: Klaus Schierbaum (schierb@uni-duesseldorf.de)
}

Received: 2 February 2016 - Revised: 10 May 2016 - Accepted: 8 June 2016 - Published: 13 July 2016

\begin{abstract}
We have developed a preparation technique to form graphite $/ \mathrm{TiO}_{2} / \mathrm{Ti}$ and platinum-graphite/ $/ \mathrm{TiO}_{2} / \mathrm{Ti}$ solid-state sensors. It is based on plasma electrolytic oxidation (PEO) of titanium foils, whereby a porous titanium oxide layer is formed with well-defined phase composition and a reproducible microstructure. A printing method is used to deposit graphite or catalytically active graphite-platinum electrodes. Our design enables the application of a variety of different detection principles such as calorimetry, impedances and amperometry. This study reports results for $\mathrm{H}_{2}, \mathrm{H}_{2} \mathrm{O}$, and $\mathrm{CO}$ sensing at room temperature with the most appropriate detection principle, electrode composition and titanium oxide microstructure.
\end{abstract}

\section{Introduction}

Very few of the gas sensors currently on the market or predicted to enter the gas sensing market soon are operable without additional heating elements. A well-known exception with widespread application is the electrochemical sensing principle. In contrast, solid-state devices whose detection principle relies mostly on catalysed molecule-surface reactions with specific target gases are typically operated with an internal heating element, capable of heating the sensor up to several hundred ${ }^{\circ} \mathrm{C}$. Figure 1a-e show schematics of different selected types of sensors, including semiconducting sensors, pellistors, field-effect devices, Schottky diodes and capacitance sensors. The number of sensing technologies here is limited, as a high technology readiness level is taken as a prerequisite. The devices shown in Fig. 1a-d operate at elevated sensing surface temperatures. One device capable of non-heated operation is the capacitance-type humidity sensor. It is based on absorption of water molecules within a polymeric network and the resulting changes to the dielectric coefficient (Farahani et al., 2014). The liquid-state electrochemical sensing principle, well known for decades (Bakker and Telting-Diaz, 2002) and typically applied in sensors for portable applications, is sketched in Fig. If for comparison. Such devices are available for a broad variety of electrochemically accessible molecules, such as carbon monox- ide. In this case, $\mathrm{CO}$ is oxidized at the working electrode (WE, i.e. sensing electrode) which is biased at low or zero voltages with respect to the reference electrode and is typically composed of graphite and a catalytically active metal. The half-cell reactions include oxidation $\left(\mathrm{CO}+\mathrm{H}_{2} \mathrm{O} \stackrel{\mathrm{WE}}{\longrightarrow}\right.$ $\left.\mathrm{CO}_{2}+2 \mathrm{H}^{+}+2 \mathrm{e}^{-}\right)$and reduction at the counter electrode $\mathrm{CE}$ $\left(\frac{1}{2} \mathrm{O}_{2}+2 \mathrm{H}^{+}+2 \mathrm{e}^{-} \stackrel{\mathrm{CE}}{\longrightarrow} \mathrm{H}_{2} \mathrm{O}\right)$ and hence involve $\mathrm{H}_{2} \mathrm{O}$ and $\mathrm{H}^{+}$ ions. It is thus contrasted with the high-temperature CO-to$\mathrm{CO}_{2}$ oxidation that occurs at the surfaces of solid-state sensors. They involve other elementary reaction steps (Bârsan and Weimar, 2002). For electrochemical sensors, the enclosure and outer and internal membranes, chosen with respect to permeation properties for $\mathrm{CO}, \mathrm{O}_{2}$ and $\mathrm{H}_{2} \mathrm{O}$, maintain the $\mathrm{H}^{+}$conducting phase aqueously for the lifetime. This is evidently unnecessary and impossible for heated solid-state sensors.

Our conceptual guideline for selecting appropriate materials for the non-heated solid-state device shown in Fig. 1g was to combine an oxide with $\mathrm{H}^{+}$ion surface mobility and water absorption capability with catalytically active metals, dispersed in the graphite top electrode. In this device, both the sensitive electrode and the oxide are accessible to gases. For this purpose, we have developed a sensor preparation technique based on plasma electrolytic oxidation of titanium (Schierbaum and El Achhab, 2011) and a printing 


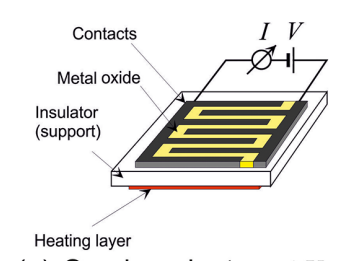

(a) Semiconductor: $\Delta V, n$

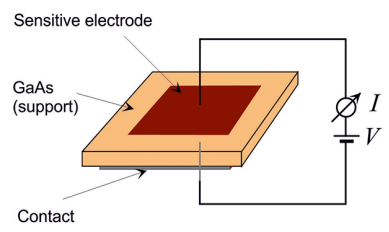

(d) Schottkydiode : $\Phi, m$

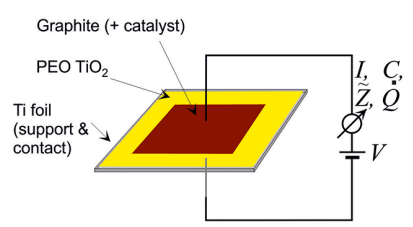

(g) PEO sensor

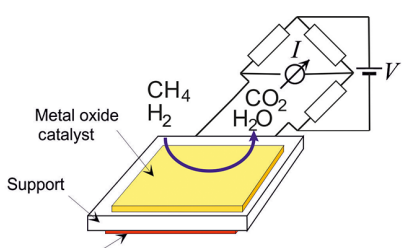

Heating layer (b) Pellistor : $\dot{Q}$

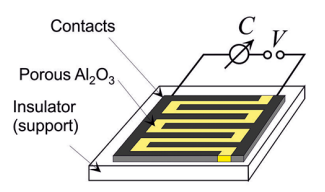

(e) Capacitor : $P$

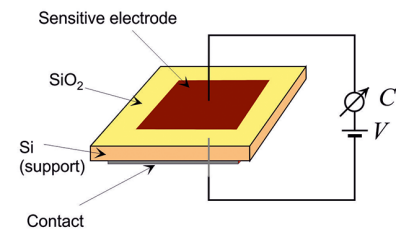

(c) FET, MOS : $\Phi$

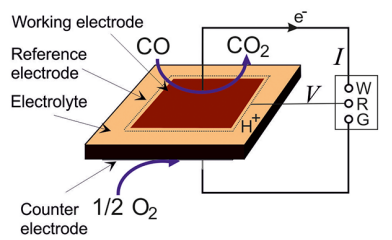

(f) ECS : I

\footnotetext{
a) Semiconducting gas sensor: $\Delta V=$ intergrain barrier, $n=$ electron density

b) Pellistor: heat production $\dot{Q}$

c) Field effect transistor, metal-oxide-semiconductor capacitor: $\Phi=$ work function

d) Schotty diode sensor: $\Phi=$ work function, $m=$ ideality factor

e) Capacitance sensor: Polarisation $P$

f) Liquid electrolyte sensor $\left(\mathrm{H}^{+}\right.$conducting electrolyte, different catalytically active electrodes, membranes not shown): $I=$ current

g) PEO sensor: Current $I$, capacitance $C$, impedance $\widetilde{Z}$, or heat production $\dot{Q}$ is measured, depending on target gas.
}

Figure 1. Schematic and comparative representation of various sensor configurations. FET, MOS, ECS and PEO denote field-effect transistor, metal-oxide semiconductor, electrochemical sensor and plasma-electrolytic oxidation, respectively.

method for the top, catalytically active electrode, which consists of graphite and nanosized platinum. Thus, the sensor element has a platinum-graphite/ $/ \mathrm{TiO}_{2} / \mathrm{Ti}$ solid-state structure.

In this study we demonstrate that platinumgraphite/ $/ \mathrm{TiO}_{2} / \mathrm{Ti}$ devices are capable of sensing $\mathrm{CO}$ and $\mathrm{H}_{2}$ at room temperature without the need for permanently operated heaters. In a first set of experiments, we show that graphite-platinum layers show sufficient catalytic activity to enable hydrogen sensing with calorimetric devices. Second, we report on room-temperature humidity sensors, based on graphite/ $/ \mathrm{TiO}_{2} / \mathrm{Ti}$ sensors, which interact with water vapour reversibly and on a surprisingly short timescale. Here, an impedance measurement is used to gain the sensor signal from the graphite/ $\mathrm{TiO}_{2} / \mathrm{Ti}$ device. Third, we apply the amperometic principle (i.e. a current measurement at a constant voltage between the Pt-C top electrode and the $\mathrm{Ti}$ substrate) to demonstrate $\mathrm{CO}$ detection in humid air at room temperature with such devices.

\section{Experiments}

We used titanium foils (99.6\%, $125 \mu \mathrm{m}$ thick, Chempur) and a laser cutting process to yield frames with small pieces of $\mathrm{Ti}$ $\left(3.5 \times 3.5 \mathrm{~mm}^{2}\right)$ as shown in Fig. 2a. For batch fabrication of sensors, we applied an automatized plasma-electrolytic oxidation by which the titanium oxide is formed under potential control and current limitation (Schierbaum and El
Achhab, 2011). The process led to excellent adhesion and stability of the oxide layer. For the present work the current limit was $200 \mathrm{~mA}$. Two different maximum voltages were applied, $150 \mathrm{~V}$ for highly porous oxide layers and $120 \mathrm{~V}$ for less porous oxide layers. Details of the preparation procedure and resulting microstructures are given in El Achhab et al. (2014). Figure $2 b$ shows the frame after oxidation. The upper electrodes were produced with a special printing technology (Hermetic Universal 6-12, Tampoprint), which uses refined catalytically active inks. Figure $2 \mathrm{c}$ shows the imprinted frame. For humidity sensors, the ink consists of pure graphite. For $\mathrm{H}_{2}$ and $\mathrm{CO}$ sensors, we add Pt paste (Chempur 900487) to achieve a low at. \% level of platinum. The single sensors are obtained from the frame by cutting the small holding strips with a sharp blade. By doing so the oxide layer is removed locally from the edges and the Ti substrate is electrically accessible for the following bonding process. Electrical connection between substrates as well as upper electrode and socket pins or PCB pads was achieved with an epoxydie bond technology (Westbond 7200CR system D) with silver epoxy glue (Loctite 3880). Figure $2 \mathrm{~d}$ shows the final sensor, mounted on a TO39 socket (Schott Singapore, Electronic Packaging).

Figure 3 displays key parts of the test chamber, sensor and device electronics. Parts exposed to test gases were fabricated by means of stereolithography (Accura XTREME, 3D Systems), all other parts by laser sintering. The electronic 

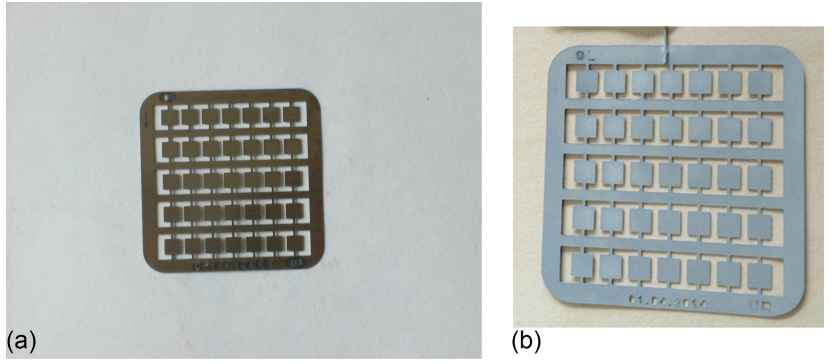

(b)
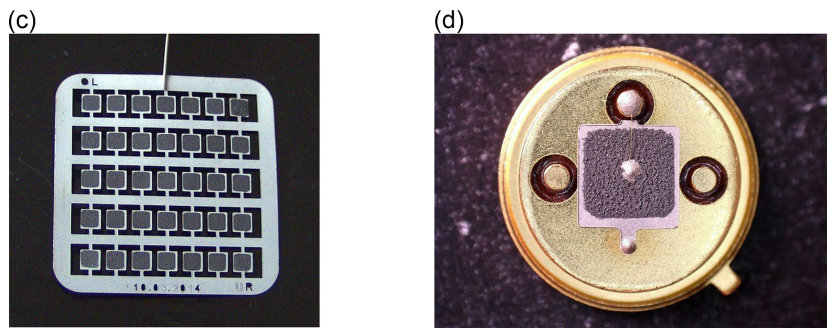

Figure 2. Key steps in the sensor fabrication process: (a) the titanium frame with 35 samples structured using a laser cutter, (b) after plasma-electrolytic oxidation of a titanium foil, (c) after printing the top electrodes and (d) after assembly of a single specimen, cut out from the processed frame, on a TO39-socket via dye-bonding. For calorimetric devices, the $\mathrm{Pt}-\mathrm{C} / \mathrm{TiO}_{2} / \mathrm{Ti}$ sample is glued to the active thermistor of the Wheatstone configuration.

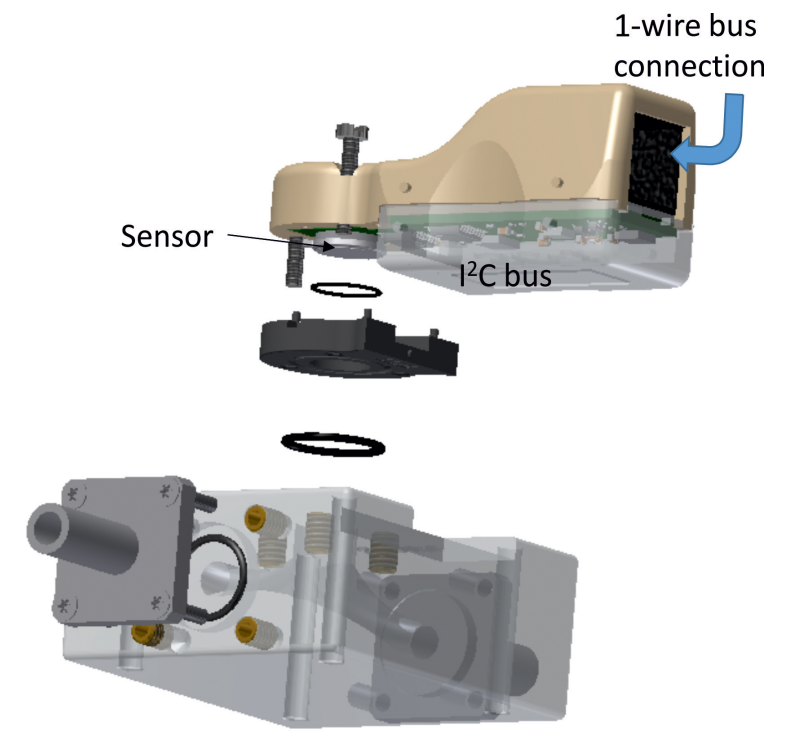

Figure 3. Test chamber with $1 / 4^{\prime \prime}$ tube gas inlet and outlet, sealing parts and device electronics with sensors mounted on a TO39 socket. The blue arrow indicates the RJ11 socket for one-wire bus connection. For internal communication, an $\mathrm{I}^{2} \mathrm{C}$ bus was used.

circuits and their layouts were developed in our department. The printed circuit boards were fabricated externally in twolayer technology with 6 mil minimum gap size.
A homemade LabVIEW code (LV 2014, National Instruments) was used for device control and data collection. A DS9490 USB adapter (Maxim Integrated) provided the connection between the one-wire network and the computer (Windows 7, 64-bit), making use of the TMEX application programming interface and corresponding drivers and libraries (see Application Note 1740, Maxim Integrated). The communication between the one-wire bus and the device electronics was established via a one-wire switch (DS2413, Maxim Integrated), which formed the SDA and SCL lines of the $\mathrm{I}^{2} \mathrm{C}$ bus, or - in the later stages of this project - via a one-wire slave-to- $\mathrm{I}^{2} \mathrm{C}$ master bridge (DS28E17, Maxim Integrated). They transmit and receive the $\mathrm{I}^{2} \mathrm{C}$ signals to and from the different components such as I/O port drivers, amplifiers, 16-bit $\Delta \Sigma$ analogue-to-digital converters (ADC), digital rheostats and potentiometers and various analogue front-end parts. For amplifiers, converters, reference voltage and current sources, integrated circuits with excellent thermal drift behaviour and ultra-low noise were chosen in tiny packages. EEPROMs were implemented to store adjustments of devices and calibration data. The one-wire bus, in which all $\mathrm{I}^{2} \mathrm{C}$ components can also be addressed individually via the unique 64-bit number stored in the onewire-to- $\mathrm{I}^{2} \mathrm{C}$ bridge, runs at normal speed $(100 \mathrm{kHz})$ or overdrive speed $(400 \mathrm{kHz}$ for the DS2413, in which the SDA data and SCL clock signals are created at its two I/O outputs, i.e. by "bit banging": Janssen, 2009). For calorimetry, a Wheatstone bridge arrangement, comprising thermistors (Murata NCP18WB473-D03RB), precision resistors and a 1024-stage digital rheostat for bridge balancing, was used in connection with a dual-precision voltage source and a 16-bit $\Delta \Sigma$-ADC with programmable gain $G\left(1 \mathrm{~V} \mathrm{~V}^{-1}\right.$ to $256 \mathrm{~V} \mathrm{~V}^{-1}$ ) and reference input, set at half the level of the bridge excitation voltage $V_{\text {bridge }}$. A full differential amplifier stage was used so the converter could swing from the negative to positive tail within an input voltage range determined by the programmed gain. For amperometry, a programmable voltage source ( -9.75 to $+9.75 \mathrm{~V}$ with 8 -bit resolution) and a high-side current amplifier with a zero-drift instrumentation amplifier with selectable gain (PGA 281, Texas Instruments) was applied. For impedance measurements, a 12-bit impedance converter (AD 5933, Analog Devices) was used together with a constant power source, set up with a power and current monitor (MAX 4210, Maxim Integrated) and controlled with a 10-bit digital-to-analogue converter. This circuit can maintain the sensor temperature slightly above the water condensation point.

\section{Results and discussion}

\subsection{Microstructure of the upper electrode}

Studies with electron microscopy (SEM), energy dispersive $\mathrm{X}$-ray spectroscopy (EDX), spatially resolved Raman X-ray diffraction and photoemission spectroscopy were previously 

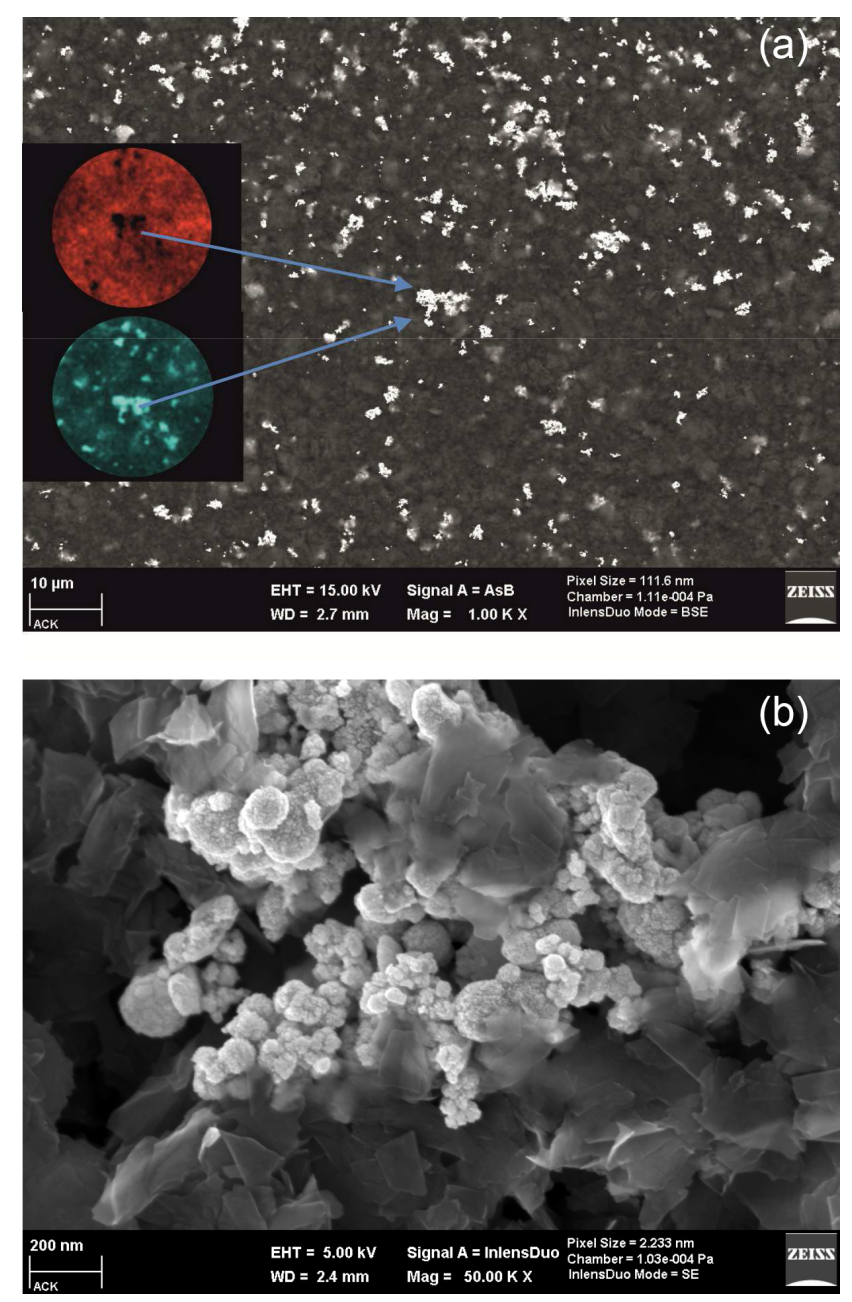

Figure 4. Scanning electron microscope image of the $\mathrm{Pt}-\mathrm{C}$ top electrode at magnifications of (a) 1000 and (b) 50000 . The insets show the $\mathrm{C}$ (red) and $\mathrm{Pt}$ (green) elemental distributions of the central Pt agglomerate, as determined with EDX.

performed to determine the relevant details of the microstructure of the titanium oxide layer (El Achhab et al., 2014). In summary, the microstructure was confirmed as consisting of a highly porous oxide which facilitates penetration of the graphite-platinum ink and explains the good adhesion of the top electrode.

Our EDX and SEM studies revealed that the nanosized Pt particles tend to agglomerate in the graphite matrices. Figure 4 displays typical SEM images at different magnifications. The bright features consist of $\mathrm{Pt}$, as confirmed with EDX. The high-resolution image shows that even the smallest Pt particles seem to consist of very small crystallites, thus creating a rough surface. The agglomerates are homogeneously distributed over the plate-like graphite matrix. Such a distribution requires a careful mixing procedure and ultrasonic treatment of the ink prior to application.
The high-resolution image of a single agglomerate of nanosized Pt particles in Fig. 4b suggests good electrical interconnectivity between $\mathrm{Pt}$ and the surrounding graphite plates. Since the upper electrode also exhibits porosity, a large three-phase boundary is formed which is accessible to the ambient gas and the sensed molecules.

\subsection{Calorimetric detection of $\mathrm{H}_{2}$ with platinum-graphite/ $/ \mathrm{TiO}_{2} / \mathrm{Ti}$ sensors}

For our first example, we chose a hydrogen sensor that uses catalytic turnover and the generated heat of oxidation (Cakabay et al., 2015). This detection principle has been known for a long time (Peinecke and Mohr, 1999) and is e.g. used in pellistors (Krawczyk and Namiesnik, 2003) and recently in calorimetric thermoelectric gas sensors (Park et al., 2014). These heated devices operate at elevated temperatures above $200{ }^{\circ} \mathrm{C}$. In contrast, the hydrogen sensor presented in this study does not require permanent heating and operates at ambient temperature. Maintaining the optimum activity of the Pt catalyst is essential to avoid strong heating of the sensor when exposed to LEL volume fractions of hydrogen in air. To achieve this, 5 at. \% Pt content was chosen for the graphite ink. In Cakabay et al. (2015) we studied the catalytic properties of the Pt particles, used in the ink, by means of an isothermally operated micro-calorimeter in a wide temperature range between 1 and $157^{\circ} \mathrm{C}$. We showed that the overall reaction rate below approximately $30^{\circ} \mathrm{C}$ is determined by the activation energy of the $\mathrm{H}_{2}$-to-water oxidation over platinum, whereas pore and film diffusion is rate-limiting above this temperature. To make use of the calorimetric operation, a Wheatstone bridge arrangement with two thermistors ( $R_{0}=47 \mathrm{k} \Omega$ at $T_{0}=25^{\circ} \mathrm{C}$, tolerance $0.5 \%$ ) was used in this study. Unlike microcalorimetry, the bridge arrangement is, however, operated without temperature regulation. The sensor foil was assembled on top of an NTC thermistor by means of a heat-conducting adhesive (TBS20S, Electrolube), while the second one remained as a reference unaffected by hydrogen exposure. We used a symmetrical Wheatstone bridge arrangement for our experiments with precision resistors and a 10-bit digital rheostat with $20 \mathrm{k} \Omega$. The arrangement is shown in Fig. 5 along with calculated voltages $V_{\text {bal }}$ as a function of the rheostat resistance $R_{\text {Rheo }}$.

$$
V_{\mathrm{bal}}=\left(\frac{R_{4}}{R_{1}+R_{4}}-\frac{R_{3}+\left\{R_{\text {Rheo }}^{-1}+R_{5}^{-1}\right\}^{-1}}{R_{3}+\left\{R_{\text {Rheo }}^{-1}+R_{5}^{-1}\right\}^{-1}+R_{2}}\right) \cdot V_{\text {bridge }}
$$

In our case the bridge voltage $V_{\text {bridge }}$ equals $4.096 \mathrm{~V}$ and is generated with a precision reference source. The temperature dependence of the thermistors' resistances is given by $R_{2}=R_{1}=R_{0} \cdot \exp \left\{B\left(T^{-1}-T_{0}^{-1}\right)\right\}$ with $B=$ $4030 \mathrm{~K}^{-1} \pm 0.5 \%$ at $T_{0}$ (Steinhart and Hart, 1968). The curves intercept at zero out-of-balance voltage. Hence, the lowest possible influence of ambient temperature changes is 


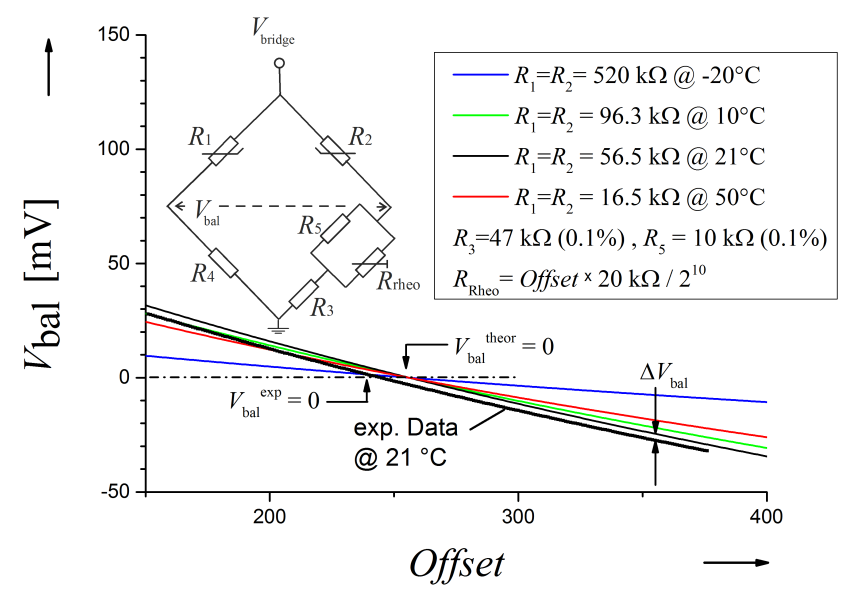

Figure 5. Calculated out-of-balance voltage $V_{\text {bal }}$ as a function of the resistance of the 10-bit digital rheostat (as given by the expression $R_{\text {Rheo }}=$ Offset $\times 20 \mathrm{k} \Omega / 2^{10}$ ) for different temperatures. $R_{1}$ and $R_{2}$ denote the active and reference thermistor, respectively. Experimental data of $V_{\mathrm{bal}} \mathrm{vs}$. Offset at $21^{\circ} \mathrm{C}$ are represented by dots and indicate a constant difference $\Delta V_{\text {bal }}$ with respect to the theoretical curve (black). Further details are given in the text.

expected for a fully balanced bridge. Experimental data are shown for $21^{\circ} \mathrm{C}$ operation of the bridge; the small difference voltage $\Delta V_{\text {bal }}$ results from additional voltage offset contributions in the electronics. Hence, the rheostat must be adjusted at a slightly different offset than calculated (compare $V_{\text {bal }}^{\text {exp }}$ and $V_{\text {bal }}^{\text {theor }}$ in Fig. 5). The deviation may have different physical origins. Fabrication tolerances of the thermistors lead to differences in their resistances and $B$ values. Similarly, thermovoltages produced in various components and soldering joints of the PCB may contribute as well. Since all these contributions are difficult to localize and compensate for (e.g. by using split resistors to reduce thermovoltage effects) under the constraints of a manageable design effort, we used the temperature sensor implemented in the $\Delta \Sigma$-ADC and readjusted the rheostat in the Wheatstone bridge through software control periodically. Thus, influences of ambient temperature changes can be further reduced.

Hydrogen exposure and the resulting heat of oxidation, produced at the sensor foil, led to temperature differences between the active and reference thermistors. This in turns yields a pronounced out-of-balance voltage. The $\Delta \Sigma$ analogue-to-digital converter used to determine the temperature difference features a special zero-baseline drift technology that guarantees a constant baseline over time. Because water vapour produces no thermal effect at the catalyst, the baseline is also stable upon changes in humidity. To reduce the inherent thermal cross-sensitivity of such a thermistorbased sensor design, appropriate shielding against airflow and infrared radiation is, however, essential.

Figure 6 displays a typical transient of the out-of-balance voltage at $23^{\circ} \mathrm{C}$, derived at an amplification of $8 \mathrm{~V} \mathrm{~V}^{-1}$ of

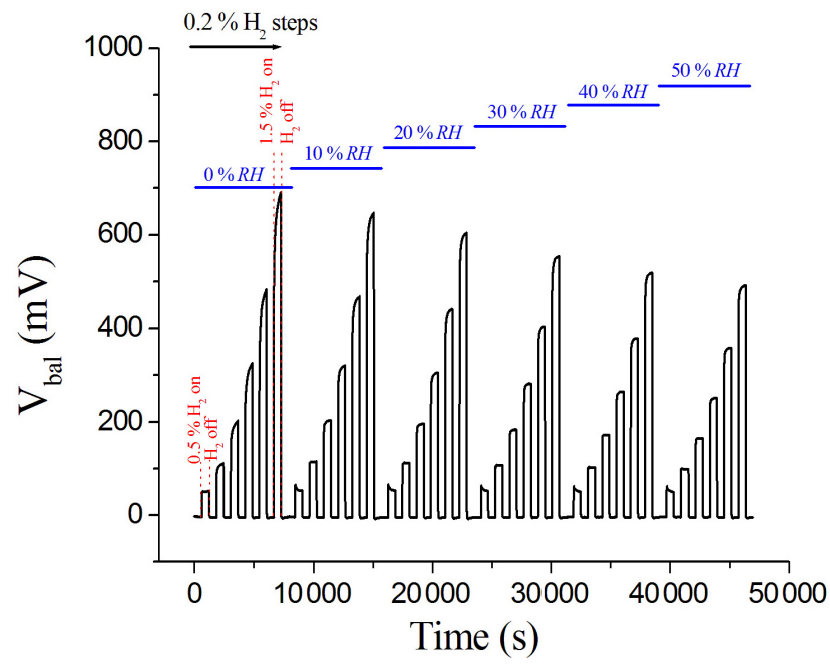

Figure 6. Typical transient of signals (out-of-balance voltage $V_{\mathrm{bal}}$ ) for hydrogen detection in dry air with Pt-graphite/ $/ \mathrm{TiO}_{2} / \mathrm{T}$ sensors at $21^{\circ} \mathrm{C}$ between 0.5 and $1.5 \mathrm{vol} \%$ (increase per step is $0.2 \mathrm{vol} \%$ ) and at different humidities from $0 \mathrm{RH}$ to $50 \% \mathrm{RH}$.

the $\Delta \Sigma$-ADC. The curve shows the response towards $\mathrm{H}_{2}$ exposures in a range from 0.5 to $1.5 \mathrm{vol} \%$, i.e. well below the low-explosive limit of $4.0 \mathrm{vol} \%$ for hydrogen in air in dry and moist air of increasing humidity (10 to $50 \% \mathrm{RH})$. Short rise and decay times within seconds are achievable. Sensitivity becomes smaller at increasing humidity mainly because of the reduced hydrogen-to-water oxidation rate. Long-term exposure experiments showed that steady-state signals are maintained. They resulted from stationary temperature differences between the active and reference thermistor, given by the different heat source and sink contributions of our setup. Up-to-the-air wiring of thermistors is found to provide larger signals, but soldering on a printed circuit board is a technologically more relevant approach for a practical implementation of the calorimetric detection principle. Tuning the thermal decoupling of both thermistors while maintaining their correlated dependency on ambient temperature changes and the dynamics upon temperature changes are essential for future achievements. In summary, the data in Fig. 6 confirm, however, the applicability of room-temperature calorimetric operation at relevant concentrations of hydrogen below LEL. Moreover, the calibration curve (out-of-balance voltage vs. $\mathrm{H}_{2}$ volume fraction) follows a linear relationship in a range between 0.5 and $1.5 \mathrm{vol} \%$ at constant temperatures. Calibration curves, however, depend on the ambient air temperature because the hydrogen oxidation is a thermally activated reaction. Also, pore diffusion may come into play at elevated temperatures (compare results for pure $\mathrm{Pt}$ in Cakabay et al., 2015). Compensation of such effects could be achieved, in principle, by taking into account data from the on-board temperature sensor in the $\Delta \Sigma$-ADC. 
We also implemented an SMD resistor underneath the $\mathrm{Ti}$ foil to briefly heat the sensor and thermally "refresh" the catalyst for different applications. According to our practical tests in a laboratory context, the typical period for such a treatment is $24 \mathrm{~h}$ with a duration of $5 \mathrm{~min}$. This procedure, which requires additional power of $500 \mathrm{~mW}$ from the USB port, is controlled by software and tracked in the EEPROM.

\subsection{Impedance metric detection of humidity with graphite/ $\mathrm{TiO}_{2} / \mathrm{Ti}$ sensors}

Most resistive-type humidity sensors use interdigitated electrodes and a humidity sensitive coating which is either made from electrolytic conductive polymers such as salts and acids or a doped ceramic sensing film (compare Fig. 1e). Typically, the resistance change follows an inverse exponential relationship with humidity and varies between approximately $1 \mathrm{k} \Omega$ and $100 \mathrm{M} \Omega$ between 20 and $90 \mathrm{RH} \%$ and response times in the range of 10 to $30 \mathrm{~s}$. The commonly applied configuration of capacitive humidity sensors is a sandwiched structure with a dielectric polymer film deposited between two electrodes. For a comprehensive review, see Farahani et al. (2014).

PEO sensors with a pure graphite electrode were used as humidity sensors. In contrast to the hydrogen sensor tests, which were performed in a gas flow system, we used a completely different procedure for tests of humidity effects on sensor signals. The sensor was quickly introduced between Erlenmeyer flasks of $200 \mathrm{~mL}$ volume with constant relative humidity, adjusted by using aqueous saturated salt solutions. We used $\mathrm{NaOH}(7 \% \mathrm{RH}), \mathrm{Mg}\left(\mathrm{NO}_{3}\right)_{2} \cdot 6 \mathrm{H}_{2} \mathrm{O}(33 \% \mathrm{RH})$, $\mathrm{MgCl}_{2} \cdot 6 \mathrm{H}_{2} \mathrm{O}(53 \% \mathrm{RH}), \mathrm{NaCl}(75 \% \mathrm{RH})$, and $\mathrm{K}_{2} \mathrm{SO}_{4}$ (97\% RH) (Pennig, 2013). A rubber stopper, through which the electrical connections ran, enclosed the interior of the vessel from the surrounding atmosphere. It should be noted that the relative humidity over the salt solution does only slightly vary with temperature, while the water partial pressure shows the same dependency on temperature than the absolute water saturation pressure does. Figure 7 shows a typical result of frequency-dependent phase angles of the impedance, measured in a frequency scan between 3 and $51.8 \mathrm{kHz}$ and calculated on-line from the real ( $\mathrm{Re})$ and imaginary (Im) data registers of the impedance converter chip. It is implemented in the sensor electronics that has the same form factor and digital communication as the hydrogen sensor. The converter has an integrated digital synthesizer that produces a sine wave with an adjustable frequency with a maximum peak-to-peak voltage of $2 \mathrm{~V}_{p-p}$ and a DC bias of $1.48 \mathrm{~V}$. Phase angles $\phi=\tan ^{-1}[\mathrm{Im} / \mathrm{Re}]$ were referenced to $\phi_{0}=\tan ^{-1}[\mathrm{Im} / \mathrm{Re}]$ that was measured with a $100,0 \mathrm{k} \Omega$ resistor. Values $\varphi-\varphi_{0}=0^{\circ}$ hence represent an resistor-type behaviour of graphite $/ \mathrm{TiO}_{2} / \mathrm{Ti}$ for the specific frequency and humidity, while $\varphi-\varphi_{0}=-90^{\circ}$ is associated with a capacitortype behaviour. It is clear from an inspection of Fig. 7 that graphite $/ \mathrm{TiO}_{2} / \mathrm{Ti}$ behaves almost like a pure capacitance in dry air in the low-frequency region and almost like a pure re-

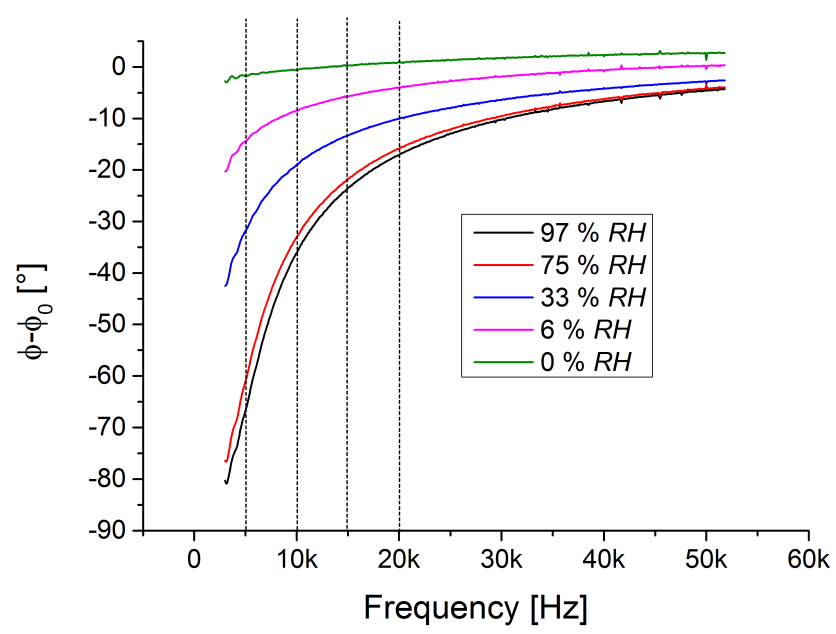

Figure 7. Typical frequency-dependent phase angles $\varphi-\varphi_{0}$ of the impedance of graphite/ $/ \mathrm{TiO}_{2} / \mathrm{Ti}$ sensors for different humidities between 3 and $51.8 \mathrm{kHz}$. Excitation voltages equal $2 \mathrm{~V}_{p-p}$. Values of $\varphi-\varphi_{0}$ are given with respect to phase angles $\varphi_{0}$ of a $100.0 \mathrm{k} \Omega$ resistor that is used instead of the sensor prior to the exposure experiment. The experiments were performed at $T=22^{\circ} \mathrm{C}$. The dotted line refers to a fixed frequency of $5 \mathrm{kHz}$ at which transients of $\varphi-\varphi_{0}$ were recorded.

sistor in humid air at $97 \% \mathrm{RH}$. The phase angle difference is associated with the phasor in the $\operatorname{Im}(\widetilde{Z})$ vs. $\operatorname{Re}(\widetilde{Z})$ plane and its change is hence related to the dielectric property of the porous $\mathrm{TiO}_{2}$ layer and how it is affected by water absorption.

The calibration curve of sensor signals $\varphi-\varphi_{0}$ as a function of relative humidity at constant frequency can be determined from Fig. 7. Here values $\varphi-\varphi_{0}$ are taken at constant frequency of $5 \mathrm{kHz}$ (dotted line). The result is shown in Fig. 8. The calibration curve was fitted with a polynomial curve of type $\phi-\phi_{0}=a+b \times(\% \mathrm{RH})+c(\% \mathrm{RH})^{2}$ (solid line) with a coefficient of determination $R^{2}$ of $0.98\left(a=-81,53^{\circ}, b=1,10\right.$ and $\left.c=-0,0028 /^{\circ}\right)$. The inserted image displays the transient of the signal during a humidity from $75 \% \mathrm{RH}$ to $33 \%$ RH in comparison with a HDC1000 humidity sensor which was integrated in our electronics. We did not calibrate the HDC 1000 and recorded the raw data from the internal registers of this device. The experiment demonstrated a faster response time of the graphite/ $/ \mathrm{TiO}_{2} / \mathrm{Ti}$ sensor (denoted as PEO in the image) in comparison with the HDC1000. It should be noted that the entire equilibration times of both sensors are identical (over $24 \mathrm{~h}$ ) and are determined by re-adjusting the humidity over the salt solution, which is disturbed by inserting the sensors in the flask. Also, the limited control of room temperature over time led to a rather large error bar of the water partial pressure as seen in Fig. 8.

Unlike the HDC1000 sensor, we noticed that the PEO sensor followed the humidity changes in breathed air during inhalation and exhalation, also with good time resolution. We ascribe the dynamics of the graphite/ $/ \mathrm{TiO}_{2} / \mathrm{Ti}$ sensor to the 


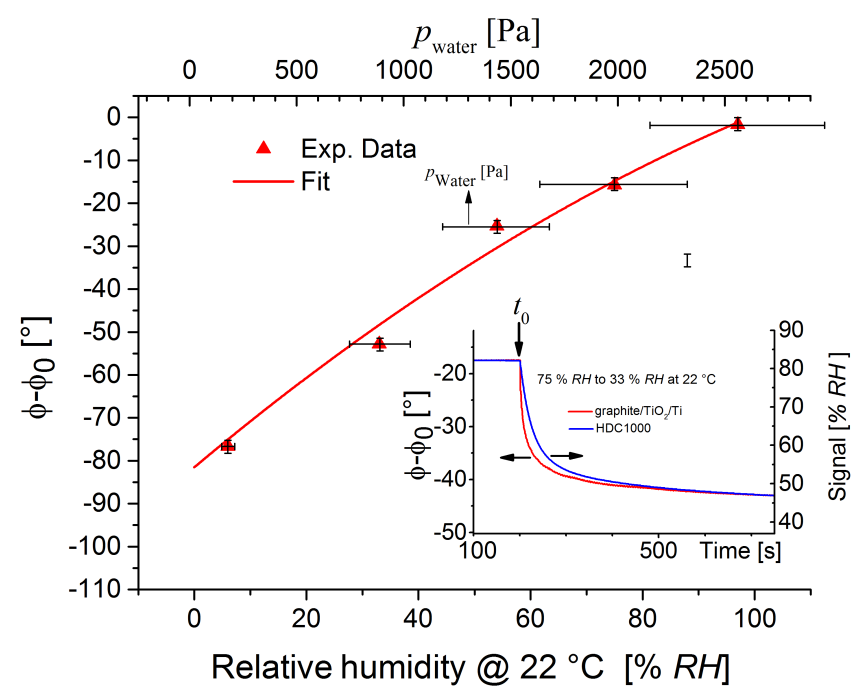

Figure 8. Sensor signals $\varphi-\varphi_{0}$ of graphite/ $\mathrm{TiO}_{2} / \mathrm{Ti}$ as a function of relative humidity and water partial pressure $p_{\text {water }}$ at $T=22^{\circ} \mathrm{C}$. Measurement was made at a constant frequency of $5 \mathrm{kHz}$ of the excitation AC voltage (experimental data: points; fit curve: solid lines). The horizontal error bars show the calculated change of the water partial pressure $p_{\text {water }}$ for a temperature change of $\pm 3{ }^{\circ} \mathrm{C}$. Note that corresponding changes in the relative humidity are below $\pm 1 \% \mathrm{RH}$ over the saturated salt solutions in the flask. The inserted image shows the transient of sensor signals of graphite/ $/ \mathrm{TiO}_{2} / \mathrm{Ti}$ in comparison with a commercial HDC1000 humidity sensor (Texas Instruments) for a humidity change from $75 \%$ to $33 \%$. At $t_{0}$, sensors were quickly extracted from a flask with $75 \% \mathrm{RH}$ and inserted into a flask with $33 \% \mathrm{RH}$.

porosity of the titanium dioxide and permeation of water molecules through the graphite top electrode.

A constant power source, digitally controllable, drove an SMD resistor underneath the sensor. This made it possible to adjust the operation temperature at different values between room temperature and $43{ }^{\circ} \mathrm{C}$ while the sensor remained in the glass vessel of a specific constant relative humidity. It should be noted that the absolute humidity (e.g. expressed by the water partial pressure, compare the top scale in Fig. 7) did not change by heating the sensor. We found that phase angles $\varphi-\varphi_{0}$ did not alter when the temperature was increased from room temperature to $43^{\circ} \mathrm{C}$. These findings suggest that the graphite/ $\mathrm{TiO}_{2} / \mathrm{Ti}$ sensor measures the absolute humidity rather than the relative humidity. The physics and chemistry of the water interaction with titanium oxide layers, produced by plasma-electrochemical oxidation, have been reported in Cakabay et al. (2016) and may serve as a starting point for an advanced understanding of humidity sensing with graphite/ $/ \mathrm{TiO}_{2} / \mathrm{Ti}$ structures.

\subsection{Amperometric detection of $\mathrm{CO}$ with Pt-graphite/ $/ \mathrm{TiO}_{2} / \mathrm{Ti}$ Sensors}

In a further set of experiments, we investigated the capability of PEO sensors for CO detection at room temperature. Figure 9a displays a typical result from an amperometric PEO sensor, operated at a bias voltage of $10 \mathrm{~V}$ at the $\mathrm{Pt}-\mathrm{C}$ top electrode, referenced to the Ti substrate. For this measurement, a small digital electronics system with adjustable precision voltage and a programmable gain amplifier was used. It measured the voltage decay over a precision $10.0 \Omega$ resistor caused by the current $I$. It is obvious from the comparison of the data for $20 \% \mathrm{RH}, 30 \% \mathrm{RH}$, and $40 \% \mathrm{RH}$, that the presence of $\mathrm{H}_{2} \mathrm{O}$ increased $\mathrm{CO}$ detection sensitivity. We determine sensor signals of $0.43 \mu \mathrm{A}$ at $20 \% \mathrm{RH}, 1.18 \mu \mathrm{A}$ at $30 \%$ $\mathrm{RH}$, and $3.17 \mu \mathrm{A}$ at $40 \% \mathrm{RH}$ in pure air, whereas the signals increase by a factor of $2.51(9.66)$ at $20 \% \mathrm{RH}, 4.74$ (28.12) at $30 \% \mathrm{RH}$, and $8.17(66.37)$ at $40 \% \mathrm{RH}$ in the presence of $50 \mathrm{ppm}(1000 \mathrm{ppm}) \mathrm{CO}$. The findings confirm the applicability of the solid-state sensor, revealed in Fig. 1g. Note that the maximum voltage, applied during the plasma-electrolytic oxidation, and hence the porosity, influence sensitivity. While the result shown in Fig. 7 was obtained for very porous titanium oxide layers (150 V, compare El Achhab et al., 2014), less porous layers can be applied to monitor even small concentrations of $\mathrm{CO}$ well below the threshold limit value of $30 \mathrm{ppm}$ in air.

Figure 10 shows results in humid air (20 to $50 \% \mathrm{RH})$ for a sensor with a titanium oxide layer prepared at $120 \mathrm{~V}$. Here, a lower bias voltage of $2 \mathrm{~V}$ was applied to keep the current through the device below $20 \mathrm{~mA}$. The baseline shift due to a humidity change from 20 to $40 \% \mathrm{RH}$ corresponds to approximately $10 \mathrm{ppm} \mathrm{CO}$. At constant humidity, the lowest detection limits are potentially in the range of $1 \mathrm{ppm}$. Furthermore, we found that the transient $I$ in the recovery regime after the CO exposure period shows an "undershooting" effect in which the current falls temporarily below the initial current $I_{0}$ prior to the $\mathrm{CO}$ exposure. It takes a period of approximately $20 \mathrm{~min}$ at a gas flow rate of $20 \mathrm{~mL} \mathrm{~s}^{-1}$ until the current again approaches $I_{0}$.

Apart from the obvious potential that PEO sensors provide for room-temperature detection of $\mathrm{CO}$, the influence of humidity is an interesting feature. At certain levels of relative humidity, surface hydroxyl groups and multi-layer $\mathrm{H}_{2} \mathrm{O}$ adsorption are known features of water chemistry on $\mathrm{TiO}_{2}$ surfaces at room temperature, depending on surface crystallography and defects (Huang et al., 2014). Furthermore, porosity favours water absorption because of the thermodynamically driven capillary effect. The diffusion of hydrogen is an intrinsic property of $\mathrm{TiO}_{2}$ and has been recently clarified with scanning tunneling microscopy (Zhang et al., 2006). We believe that $\mathrm{CO}$-to- $\mathrm{CO}_{2}$ conversion, which has also been experimentally confirmed from the rising $\mathrm{CO}_{2}$ concentration in the downward airflow behind the sensor, can be associated with the presence of surface hydroxyl groups and $\mathrm{H}^{+}$ion surface 

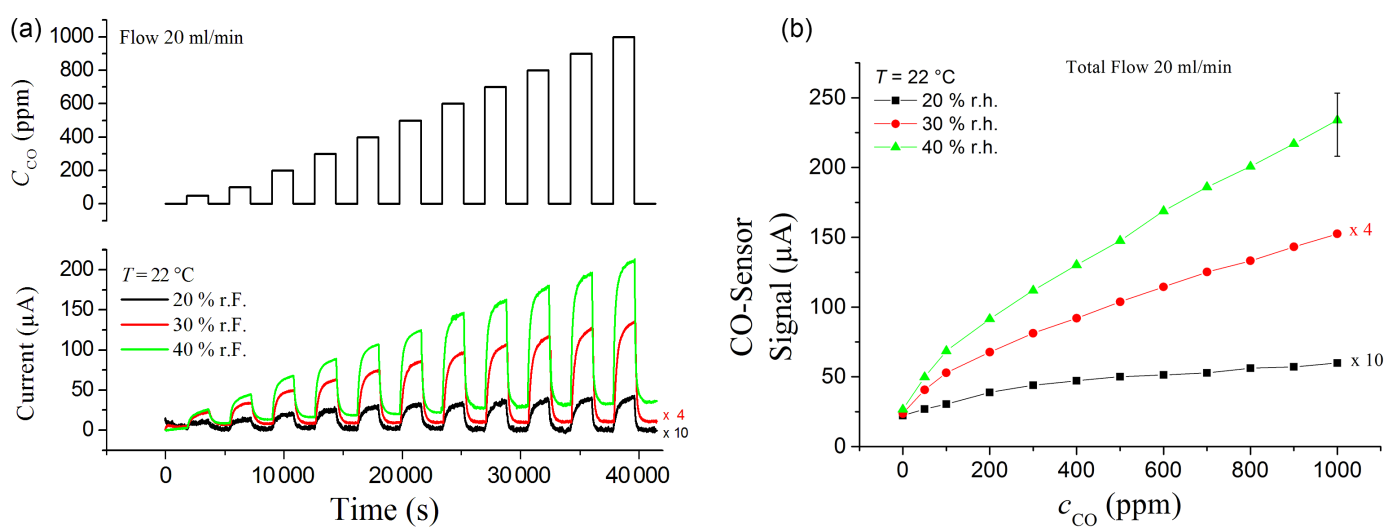

Figure 9. (a) Typical transients of signals of $\mathrm{Pt}$-graphite/150 V- $\mathrm{TiO}_{2} / \mathrm{Ti} \mathrm{CO}$ sensors at different humidities for $\mathrm{CO}$ volume fractions between 50 and $1000 \mathrm{ppm}$. The bias voltage is $10 \mathrm{~V}$. The titanium oxide layer was prepared at a maximum voltage of $150 \mathrm{~V}$. (b) Signal (here: current after 30 min exposure time) as a function of concentration. The error is mainly determined by variations of humidity in the flow set-up.

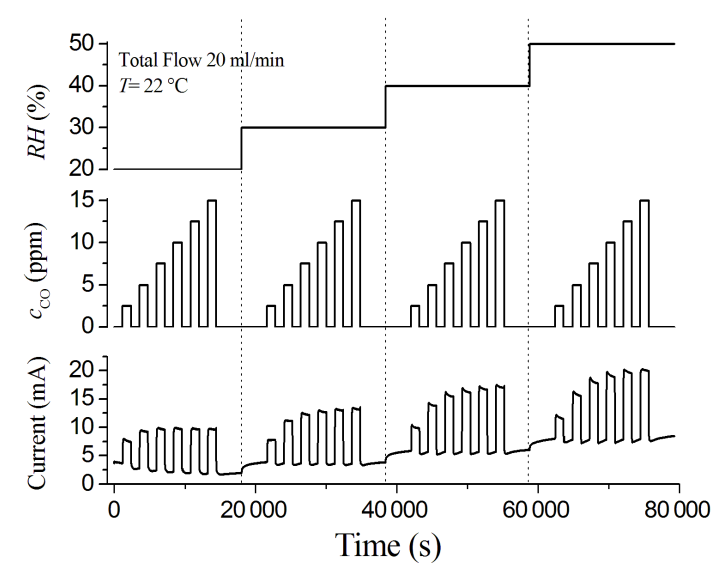

Figure 10. Typical transients of signals of Pt-graphite/120 V$\mathrm{TiO}_{2} / \mathrm{Ti} \mathrm{CO}$ sensors at 20,30, 40, and $50 \mathrm{RH} \%$ for $\mathrm{CO}$ volume fractions between 2.5 and $15 \mathrm{ppm}$. The bias voltage is $2 \mathrm{~V}$.

diffusion over $\mathrm{TiO}_{2}$ surfaces. This should be the subject of further studies. From a practical point of view, the most interesting approach would be to stabilize the relative humidity in the vicinity of the sensing element, as is done in liquid-state electrochemical sensors.

It should be noted that $\mathrm{Pt}$-graphite/ $\mathrm{TiO}_{2} / \mathrm{Ti}$ sensors for $\mathrm{CO}$ are affected by hydrogen. The interaction of $\mathrm{H}_{2}$ with $\mathrm{Pt} / \mathrm{TiO}_{2} / \mathrm{Ti}$ has been previously studied in detail (see the reference of Schierbaum and El Achhab, 2011). Also, oxidizing gases like $\mathrm{NO}_{2}$ interact with $\mathrm{Pt}$-graphite/TiO $/$ Ti. The results will be the subject of another publication.

\section{Conclusions}

We presented the sensing characteristics of solid-state devices, based on platinum-graphite/titanium oxide/titanium sandwiched structures for hydrogen, humidity and carbon monoxide detection. The titanium oxide layer is prepared by plasma electrolytic oxidation. We demonstrated the applicability of different detection principles, a calorimetric device for hydrogen, an impedance device for humidity and an amperometric device for $\mathrm{CO}$ detection. All devices operated without permanent heating. It is likely that this particular property offers an appropriate way to use polymeric membranes in the sensor enclosure to improve sensor performance under ambient air conditions.

Acknowledgements. This research was supported by the German Federal Ministry of Education and Research under project no. VIP 03V0356. Responsibility for the content of this publication lies with the authors. We thank G. Koschek and J. Ackermann (Carl Zeiss Microscopy $\mathrm{GmbH}$ ) for providing us with scanning electron microscopic images and energy-dispersive X-ray spectra.

Edited by: A. Schütze

Reviewed by: three anonymous referees

\section{References}

Bakker, E. and Telting-Diaz, M.: Electrochemical Sensors, Anal. Chem., 74, 2781-2800, 2002.

Bârsan, N. and Weimar, U.: Understanding the Fundamental Principles of Metal Oxide Based Gas Sensors; The Example of CO Sensing with $\mathrm{SnO}_{2}$ Sensors in the Presence of Humidity, J. Phys.-Condens. Mat., 15, R813-R839, 2003.

Cakabay, Ö., El Achhab, M., and Schierbaum, K.: Thermal Properties of Solid-State $\mathrm{Pt} / \mathrm{TiO}_{2} / \mathrm{Ti}$ Emf Cells Studied by Microcalorimetry, Appl. Phys. A, 118, 1127-1132, doi:10.1007/s00339-014-8927-9, 2015.

Cakabay, Ö., El Achhab, M., and Schierbaum, K.: The Effect of Water Vapour on Pt/TiO $2 /$ Ti Emf Cells, The Journal of Physical Chemistry C, 120, 9061-9067, doi:10.1021/acs.jpcc.6b01315, 2016.

El Achhab, M., Erbe, A., Koschek, G., Hamouich, R., and Schierbaum, K.: A Microstructural Study of the Structure of 
Plasma Electrolytically Oxidized Titanium Foil, Appl. Phys. A, doi:10.1007/s00339-014-8392-5, 2014.

Farahani, H., Wagiran, R., and Hamidon, M. N.: Humidity Sensors Principle, Mechanism, and Fabrication Technologies: A Comprehensive Review." Sensors, 14, 7881-7939, doi:10.3390/s140507881, 2014.

Huang, L., Gubbins, K. E., Li, L., and Lu, X.: Water on Titanium Dioxide Surface: A Revisiting by Reactive Molecular Dynamics Simulations, Langmuir, 30, 14832-14840, doi:10.1021/la5037426, 2014.

Janssen, F.: Application Note 4482, Maxim Integrated, available at: https://www.maximintegrated.com/en/app-notes/index. mvp/id/4482 (last access: 7 July 2016), Elektor, 3, 54, 2009.

Krawczyk, M. and Namiesnik, J.: Application of a Catalytic Combustion Sensor (Pellistor) for the Monitoring of the Explosiveness of a Hydrogen-Air Mixture in the Upper Explosive Limit Range, J. Autom. Method. Manag., 25, 115-122, 2003.

Park, N.-H., Akamatsu, T., Itoh, T., Izu, N., and Shin, W.: Calorimetric Thermoelectric Gas Sensor for the Detection of Hydrogen, Methane and Mixed Gases, Sensors, 14, 8350-8362, doi:10.3390/s140508350, 2014.
Pennig, A.: in: VDI-Wärmeatlas, edited by: VDI e.V., eReference, ISBN-13: 978-3-642-19981-3, Springer-Verlag Berlin Heidelberg, 609 pp., 2013.

Peinecke, V. and Mohr, P.: Sensor for Measuring the Composition of Mixtures of Hydrogen and Oxygen Gas, No. US5959190 A, 28 September 1999.

Schierbaum, K. and El Achhab, M.: Generation of an Electromotive Force by Hydrogen-to-Water Oxidation with $\mathrm{Pt}$ coated Oxidized Titanium Foils, Phys. Status Solidi A, 1-7, doi:10.1002/pssa.201127400, 2011.

Steinhart, J. S. and Hart, S. R.: Calibration curves for thermistors, Deep-Sea Res., 15, 497-503, doi:10.1016/0011-7471(68)900570,1968

Zhang, Z., Bondarchuk, O., Kay, B. D., White, J. M., and Dohnálek, Z.: Imaging Water Dissociation on $\mathrm{TiO}_{2}$ (110): Evidence for Inequivalent Geminate OH Groups, J. Phys. Chem. B, 110, 21840 21845, 2006. 